\title{
Electronic Structures of Aluminum and Aluminum Clusters Doped with Other Atoms
}

\author{
Shane J. Brennan, Carmel B. Breslin, ${ }^{*}$ and Charles M. Quinn \\ Department of Chemistry, National University of Ireland, Maynooth, ${ }^{a}$ Maynooth, County Kildare, Ireland
}

\begin{abstract}
Electronic structure calculations have been carried out for various model $\mathrm{Al}_{n} \mathrm{X}$ clusters with $\mathrm{X}$ an atom of an element known, or likely, to give rise to changes in the corrosion resistance of AIX alloys. The calculations involved the use of a novel orthogonal outer-sphere MSX $\alpha$ technique and results for the partial densities of states for the clusters $\mathrm{Al}_{50}, \mathrm{Al}_{49} \mathrm{In}, \mathrm{Al}_{49} \mathrm{Zn}, \mathrm{Al}_{49} \mathrm{Cr}, \mathrm{Al}_{49} \mathrm{Ti}_{\text {, and }} \mathrm{Al}_{49} \mathrm{Ta}$ are consistent with the hypothesis that activation of aluminum is to be expected for dopant atoms with filled d orbitals, giving rise to Friedel states at the surface, while passivation is to be expected when the d-band density is distributed about the cluster Fermi level.
\end{abstract}

\section{Introduction}

The corrosion resistance of aluminum, doped with alloying elements, in aggressive environments is known to vary with the nature of the dopant metal. In particular, passivation has been reported for $\mathrm{Cr}, \mathrm{Ti}$, or Ta as dopants ${ }^{1-4}$ and activation has been observed in aluminum samples doped with $\mathrm{Zn}$ or $\mathrm{In}^{5,6}$ The occurrence of passivation, in particular, due to doping has been associated with effects due, for example, to modifications of the $\mathrm{pH}$ of zero charge, ${ }^{2}$ variations in the solubility of the oxidized phase, ${ }^{7}$ variations in the rate of dissolution of the alloy ${ }^{1}$ and the pairing of highly charged alloying element ions and mobile cation vacancies. ${ }^{8}$

This is the first report of the results of calculations undertaken to discover whether any features of the electronic structure of doped aluminum, also, can be associated with the passivation or activation of aluminum due to the presence of dopants at the metal/oxide interface.

The calculations have been carried out using a novel orthogonal outer-sphere version of the multiple scattering $x$-alpha technique (MSX $\alpha$ ) approximation. ${ }^{9,10}$ The advantage of this method, for cluster calculations, is the straightforward manner in which all the eigenvalues of irreducible point symmetry of a cluster can be identified. Thus, densities of states and partial densities of states at surface sites can be determined over the full bandwidth of the alloys, without there being any uncertainty as to the states being counted in the densities.

\section{Methodology}

The orthogonalized outer-sphere approach to MSX $\alpha$ calculations has been used to study the changes in local and partial local densities of states at surface sites in clusters of atoms, $\mathrm{Al}_{49} \mathrm{X}$, chosen to model a doped (100) surface of an aluminum single crystal. A cluster of stoichiometry $\mathrm{Al}_{49} \mathrm{X}$ comprises the first 12 geometric orbits" of the point group, $\mathrm{C}_{4 v}$, of the in-plane surface site of the (100) face of the face-centered cubic (fcc) aluminum crystal. Given that this site is the location of a transition metal atom or a metal atom with a filled d-shell, the calculations should return $37 a_{1}, 25 b_{1}$, $25 \mathrm{~b}_{2}, 16 \mathrm{a}_{2}$, and $51 \mathrm{e}$-type eigenvalues for counting in the total density of states (DOS) data.

The DOS $\left[\rho^{c}(E)\right]$ data were constructed from the discrete results for the eigenvalues calculated as suggested by Salahub and Messmer ${ }^{12}$

$$
\rho^{c}(E)=\left(2 \pi \sigma^{2}\right)^{-1 / 2} \Sigma_{i} \exp \left[-\left(E-\epsilon_{i}^{2} / 2 \sigma^{2}\right]\right.
$$

with the Gaussian broadening parameter $(\sigma)$ set to 0.05 , which value corresponds to a full width at half maximum (FWHM) of $1.6 \mathrm{eV}$ in the Gaussians. Local density of states (LDOS) data follow when this relation is modulated by the square of the wavefunction component at the local site. Partial local densities (PLDOS) are obtained when wavefunction components locally are counted by spherical harmonic type. In the case of the orthogonalized MSX $\alpha$ method there is only the intersphere wavefunction to be partitioned among the muffin-tin sites since the outer-sphere regional functions are of zero amplitude for the states of interest and these parameters can be estimated straightforwardly.

\section{Results and Discussion}

In Fig. 1, the LDOS data for the $\mathrm{Al}_{49} \mathrm{X}$ clusters are presented with respect to the Fermi level energy of each cluster. For indium and

\footnotetext{
* Electrochemical Society Active Member.
}

a Formerly St. Patrick's College. zinc-doped clusters the $\mathrm{sp}$ band is that found for the $\mathrm{Al}_{50}$ cluster and the $d$-levels are discrete and unmixed. For chromium, tantalum, and titanium-doped clusters the difference in the DOS data is the strong mixing of the d-levels about the cluster Fermi level. This effect apart, the sp bands are reasonable representations of the free-electron dispersion curve expected for $\mathrm{Al}$ at moderate energies. ${ }^{13,14}$

In Fig. 2, the partial LDOS data for the $\mathrm{Al}_{49} \mathrm{X}$ clusters are presented for the d-levels of the $X$ atom. It is clear that there is the division suggested for the dopant atom d-levels depending on whether these levels are partially or fully occupied. Friedel-like states are found when the d-levels are occupied or almost fully occupied and this includes the cases of In and Zn. Strong coupling of the d-levels with the $s p$ band at the Fermi level is found when the d-levels of the $X$ atom are partially occupied, $\mathrm{Cr}, \mathrm{Ta}$, and $\mathrm{Ti}$.

For bulk sites, doping of aluminum and other simple metals with transition metal atoms has been recognized and shown to give rise to Friedel bound states ${ }^{\dagger 4-18}$ at dopant atoms. For dopant atoms with partially filled d-orbital counted in the electron configurations, the densities of states around the Fermi levels of the alloys are increased. For dopants, with filled $(n-1)$ d-shells narrow Lorentzian features are found in the DOS data. ${ }^{19,20}$

The present results for surface sites give rise to the same effects as are found in the bulk. But, moreover, there appears to be some correlation with the activation or passivation behavior of the dopants and local surface electronic structure. It would seem that more mixing of the d-levels at the Fermi level can be associated with increasing passivation $(\mathrm{Cr}, \mathrm{Ta}, \mathrm{Ti})$, while the greater the discreteness of the Friedel state due to the d-levels the greater the activation found for the dopant atom $(\mathrm{Zn}, \mathrm{In})$.

The greater mixing of the d-levels at the Fermi level result in more extensive bonding at the doped surface sites in the $\mathrm{Al}_{49} \mathrm{X}$ cluster. This more efficient bonding extends also into the oxide layer which develops on these clusters. But, it seems that the greater bonding in the substrate alloy phase is more important in the localized dissolution of binary AIX alloys, where $X$ represents a passivating element, as shown by Frankel et al.' from pitting potential and repassivation potential measurements on AICr, AIMo, and AINb alloys. The greater bonding in the surface oxide films may lead to higher pitting potentials but the excellent corrosion resistance afforded by these materials seems to be related more to the dissolution of the substrate alloy phase. The enhanced bonding in the AIX alloy leads to an ennoblement of the dissolution process and the requirement of higher potentials to achieve a given current density and maintain active dissolution conditions. This notion is supported also by the fact that tungsten is depleted from the passive films formed on AIW alloys yet tungsten is extremely effective in promoting passivation of aluminum. ${ }^{4}$ This theory accounts also for the proportionality between the concentration of the dopant and the resistance to localized dissolution observed experimentally' as the sphere of influence of the dopant depends on the dopant concentration.

The development of discrete Friedel states, as found for the $\mathrm{Al}_{49} \mathrm{In}$ and $A l_{49} \mathrm{Zn}$ clusters, may give rise to increased repulsive interactions locally because of the bound d-electron density and so to accelerated dissolution and activation of aluminum. Again, this local situation may decrease the cohesion of the oxide film locally. Accelerated dissolution of the alloy phase is consistent with the experimental observations, ${ }^{5.6}$ but also, incomplete formation of a protective oxide layer could account for such experimental observations.

Manuscript received Feb. 26, 1997.

The National University of Ireland, Maynooth, assisted in meeting the publication costs of this article. 
Fig. 1. Total densities of states for the $\mathrm{Al}_{49} \mathrm{X}$ clusters. Note that the only substantial differences are to be seen in the occurrences of d-level contributions. Energies are given in Rydbergs with respect to the normalized Fermi level in each case: $(\mathbb{C})$ $\mathrm{Al}_{50},(*) \mathrm{Al}_{49} \mathrm{In},(\mathrm{O}) \mathrm{Al}_{48} \mathrm{Zn},(\mathbf{C})$ $\mathrm{Al}_{49} \mathrm{Cr}$, (A) $\mathrm{Al}_{49} \mathrm{Ti}$, and $(O)$ $\mathrm{Al}_{49} \mathrm{Ta}$.

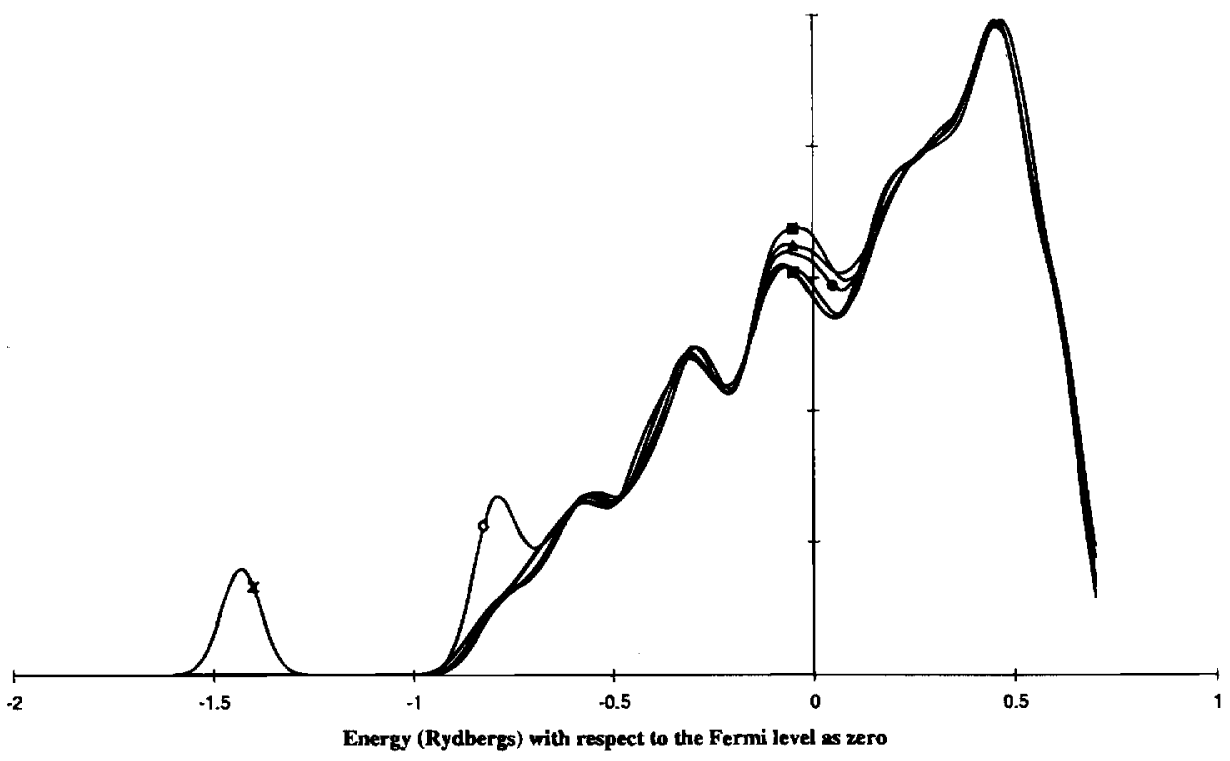

Fig. 2. The partial $d$-band local densities of states for the clusters given in the legend. Again energy is in Rydbergs and is relative to the chosen zero at the Fermi level in each cluster: $(*)$ $\mathrm{Al}_{49} \mathrm{In}$, (O) $\mathrm{Al}_{49} \mathrm{Zn},(\square) \mathrm{Al}_{49} \mathrm{Cr}$, (A) $\mathrm{Al}_{49} \mathrm{Ti}$, and $(O) \mathrm{Al}_{49} \mathrm{Ta}$.

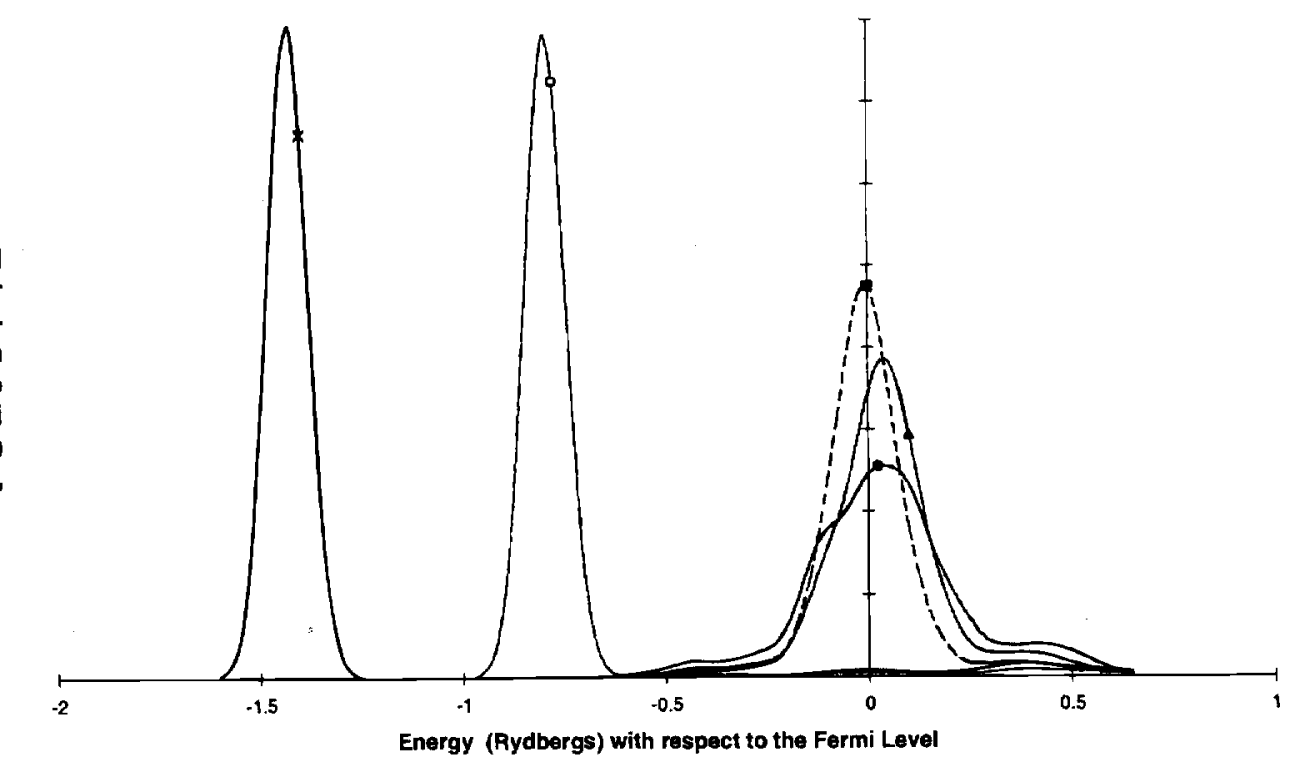

\section{REFERENCES}

1. G. S. Frankel, R. C. Newman, C. V. Jahnes, and M. A. Russak, This Journal, 140, 2192 (1993).

2. E. McCafferty, P. M. Natishan, and G. K. Hubler, Corros. Sci, 30, 209 (1990).

3. W. C. Moshier, G. D. Davis, S. Ahearn, and H. F. Hough, This Journal, 134, 2677 (1987).

4. B. A. Shaw, T. L. Fritz, G. D. Davies, and W. C. Moshier, ibid., $137,1317(1990)$.

5. J. T. Reding and J. J. Newport, Mater. Protection, 5, 15 (1966).

6. C. B. Breslin and W. M. Carroll, Corros. Sci., 34, 1099 (1993).

7. Z. Szklarska-Smialowska, ibid., 33, 1193 (1992).

8. M. Urquidi-Macdonald and D. D. Macdonald, This Journal, 136, 961 (1989).

9. S. J. Brennan and C. M. Quinn, To be submitted.
10. M. Cook and D. A. Case, Quantum Chemistry Program Exchange (QCPE), 5, 465 (1982).

11. P. W. Fowler and C. M. Quinn, Theoretica Chim. Acta, 70, 333 (1986).

12. D. R. Salahub and R. P. Messmer, Phys. Rev. B, 16, 2526 (1977).

13. V. Heine, Proc. R. Soc. London, Ser. A, 240, 361 (1957).

14. B. Segall, Phys. Rev., 124, 1797 (1961).

15. J. Friedel, Can. J. Phys., 34, 1190 (1956).

16. J. Friedel, J. Phys. Radium, 19, 573 (1958).

17. J. Friedel, Supplo. Nuovo Cimento, VII, 287 (1958)

18. P. W. Anderson, Phys. Rev., 124, 41 (1961).

19. M. Morinaga, S. Nasu, H. Adachi, J. Saito, and N. Yukawa, J. Phys.: Condens. Matter, 3, 6817 (1992).

20. P. Steiner, H. Höchst, W. Steffen, and S. Hüffner, Z. Phys., B38, 191 (1980). 